J. Austral. Math. Soc. Ser. B 29 (1988), 266-269

\title{
DETERMINING STATISTICAL DOMINANCE BY LINEAR PROGRAMMING
}

\author{
ROSS G. DRYNAN ${ }^{1}$
}

(Received 21 February 1986; revised 27 November 1986)

\begin{abstract}
This note generalises the necessary and sufficient conditions for one act to be dominated by another when the two acts available to the decision maker have outcomes contingent on discrete states of nature whose probabilities of occurrence are known only to the extent of linear partial information. The generalisation relates to the dominance of an act by a set of acts. The presentation is in terms of general vector dominance, of which statistical dominance is only a particular case.
\end{abstract}

\section{Introduction}

In a recent paper on decision making with less than full information about the probability distributions of outcomes from the various acts confronting a decision maker, Kmietowicz and Pearman [4] have suggested the use of a maximum $E$ min criterion: select that act which, in the face of linearly constrained information about probabilities, maximises guaranteed expected payoff. Later in their paper, they suggested that the criterion, together with the analogous maximum $E$ max, may be useful in establishing statistical dominance of one act over another ${ }^{2}$. In particular if act $i$ has an $E$ min (and $E$ max) greater than the $E$ max (and $E$ $\min$ ) for act $j$, then $i$ dominates $j$. That is, even without exact knowledge of the

\footnotetext{
'Department of Agriculture, University of Queensland. St. Lucia, Queensland 4067, Australia.

${ }^{2}$ The concept of dominance in this note relates to a decision maker with imprecise knowledge of acts. This is in contrast to stochastic dominance (e.g. Fishburn [3]) where the imprecision concerns the decision maker's risk attitudes.

(c) Copyright Australian Mathematical Society 1988, Serial-fee code 0334-2700/88
} 
probabilities, $i$ is unambiguously preferred to $j$ by decision makers who assess acts on the basis of expected payoff. They state further, that if the $E$ min for $i$ is less than the $E$ max for $j$, then one cannot in general state that $i$ is preferred to $j$.

Both statements about dominance are correct: the former conditions are sufficient (though not necessary) for dominance; the latter conditions are neither sufficient nor necessary. In a subsequent paper, Kmietowicz and Pearman [5] substantially derive necessary and sufficient conditions for one act to dominate another.

In the remainder of this note, the necessary and sufficient conditions are derived more directly and in a way which generalises the statistical dominance result in two directions. First, necessary and sufficient conditions for one act to be dominated by a set of other acts are given. Second, the statistical dominance concept is shown to be only one application of a more general vector efficiency concept. The generalised necessary and sufficient conditions are simple and readily implemented.

\section{Necessary and sufficient conditions}

Consider the problem:

$$
\max _{i} Z_{l}
$$

where

$$
\begin{gathered}
Z_{t}=\min _{\mathbf{C}} \mathbf{C}^{\prime} \mathbf{X}, \\
\text { s.t. } \mathbf{A C} \leqslant \mathbf{b} \\
\mathbf{C} \geqslant \mathbf{0}
\end{gathered}
$$

where $i$ identifies one of $k$ acts, each defined by an $n$ element transposed column vector outcome $\left[X_{1}, X_{21}, \ldots, X_{n i}\right]^{\prime} ; \mathbf{A}$ and $\mathbf{b}$ are known coefficients, and $\mathbf{C}$ is an $n$-vector of imprecisely known coefficients. The problem setting here is more general than that of Kmietowicz and Pearman. Theirs is an obvious particular case in which $\mathbf{C}$ is a vector of probabilities.

The solution to the problem is that act which maximises the smallest $\mathbf{C}^{\prime} \mathbf{X}$. While the criterion offers security, it may lead to the wrong (with hindsight) decision. For act $i$ to be unambiguously preferred to $j$, the former's $\mathbf{C}^{\prime} \mathbf{X}_{i}$ must be greater than $\mathbf{C}^{\prime} \mathbf{X}$, for each possible $\mathbf{C}$ value. That is, $\mathbf{C}^{\prime}\left(\mathbf{X}_{t}-\mathbf{X}_{\jmath}\right)$ must be positive. That this is sufficient for strict dominance is obvious. That it is necessary is also clear: if $\mathbf{C}^{\prime}\left(\mathbf{X}_{i}-\mathbf{X}_{j}\right)<0$ for some $\mathbf{C}$, then $j$ is preferred to $i$ for that $\mathbf{C}$; or if $\mathbf{C}^{\prime}\left(\mathbf{X}_{t}-\mathbf{X}_{j}\right)=0$ for any $\mathbf{C}$, then neither $i$ nor $j$ is preferred to the other for this value of $\mathbf{C}$. 
To establish dominance between a pair of acts, one can use the following linear program:

$$
\begin{gathered}
\min _{\mathbf{C}} Z=\mathbf{C}^{\prime}\left(\mathbf{X}_{i}-\mathbf{X}_{j}\right) \\
\text { s.t. } \mathbf{A C} \leqslant \mathbf{b} \\
\mathbf{C} \geqslant \mathbf{0},
\end{gathered}
$$

and theck the soiution value of $Z$. If $Z \geqslant 0, i$ is efficient (i.e. not dominated). If $Z>0, j$ is dominated. But if $Z=0, j$ is also efficient. On the other hand, if $Z<0, j$ is efficient. To check if $i$ is dominated, one must solve for the maximum $\mathbf{C}^{\prime}\left(\mathbf{X}_{1}-\mathbf{X}_{j}\right)$. If this is negative, $i$ is dominated; otherwise $i$ is also efficient.

\section{Generalised dominance}

When there are $k$ acts, a generalised concept of dominance is more appropriate than the pair-wise dominance considered above ${ }^{3}$. Thus an act $i$ is dominated if there is always some other preferable act for all values of $\mathbf{C}$. An appropriate linear program for determining if $i$ is dominated is the maximin formulation:

$$
\begin{aligned}
& \underset{\mathbf{C}}{\operatorname{maximise}} Z \\
& \text { s.t. } \mathbf{A C} \leqslant \mathbf{b} \\
& \quad\left(\mathbf{X}_{\iota}-\mathbf{X}_{j}\right)^{\prime} \mathbf{C}-Z \geqslant 0 \text { for } j=1, \ldots, k ; j \neq i \\
& \mathbf{C} \geqslant \mathbf{0}
\end{aligned}
$$

If $Z \geqslant 0$, act $i$ is efficient, but if $Z<0$, then it is dominated. A series of no more than $k$ similar linear programs can be used to define the complete set of efficient acts.

The interpretation of $\mathbf{C}$ as a vector of probabilities has already been noted. Another interesting case is that where the $n$ dimensions of $\mathbf{X}_{i}$ refer to goal or objective achievements, and $\mathbf{C}$ reflects the weights to be attached to these goals. Typically in the multi-objective decision making literature (e.g. Cohon and Marks [1]) an act is said to be (Pareto) efficient if there is no other act that has a higher $\mathbf{X}$ for all dimensions. The introduction of the goal weights $\mathbf{C}$ allows some trade-offs between goals. With $\mathrm{C}$ known exactly, one can define an optimum choice. With $\mathbf{C}$ known imprecisely, one may reduce the standard Pareto efficient set only partially towards a unique optimum.

\footnotetext{
${ }^{3}$ There is a parallel concept in the literature of stochastic dominance originating in Fishburn's concept of convex stochastic dominance (Fishburn [3], Drynan [2]).
} 


\section{Acknowledgement}

This paper was written while the author was on leave as Honorary Visiting Fellow, Department of Agricultural Economics, University of Manchester.

\section{References}

[1] J. L. Cohon and D. H. Marks, "A review and evaluation of multiobjective programming techniques", Water Resources Research 11 (1975) 208-220.

[2] R. G. Drynan, "Experimentation-its value to the farm decision maker", Ph. D. thesis, University of New England, Armidale, Australia, 1977.

[3] P. C. Fishburn, "Convex stochastic dominance", J. Econom. Theory 7 (1974) 143-158.

[4] Z. W. Kmietowicz and A. D. Pearman, "Decision theory and strict ranking of probabilities", European J. Oper. Res. 9 (1982) 397-404.

[5] Z. W. Kmietowicz and A. D. Pearman, "Decision theory, linear partial information and statistical dominance", Omega 12 (1984) 391-399. 